of support provided to networks or teams by the CTSI, department or other institutional mechanism.

3415

\author{
Percent Predicted Peak Exercise Oxygen Pulse Is a \\ Marker of Cardiac Reserve Following Thoracic \\ Radiotherapy \\ Justin McNair Canada ${ }^{1}$, Elisabeth Weiss², John Grizzard², \\ Ronald Evans ${ }^{2}$, Ryan Garten², Benjamin Van Tassell ${ }^{2}$, \\ Salvatore Carbone, $\mathrm{PhD}^{2}$, Cory R. Trankle, $\mathrm{MD}^{2}$, \\ Hayley Billingsley, $\mathrm{RD}^{2}$, Dinesh Kadariya, $\mathrm{MD}^{2}$ and \\ Antonio Abbate, $\mathrm{MD}^{2}$ \\ ${ }^{1}$ VCU Center for Clinical and Translational Research and \\ ${ }^{2}$ Virgnia Commonwealth University
}

OBJECTIVES/SPECIFIC AIMS: Cardiac radiation exposure following anti-cancer (CA) thoracic radiotherapy (RT) treatment increases risk of heart failure in a dose-dependent manner with a predominantly restrictive cardiomyopathy phenotype and is characterized by a diffuse fibrosis within the myocardium. The peak oxygen pulse (O2Pulse) determined at cardiopulmonary exercise testing (CPET) is the quotient of oxygen consumption (VO2) divided by the heart rate (HR) at peak exercise. Through deduction of the Fick equation $(\mathrm{VO} 2=$ cardiac output $(\mathrm{CO}) \mathrm{x}$ arteriovenous oxygen difference) it provides a noninvasive estimate of the stroke volume response to exercise. Knowledge of the relationship between cardiac radiation dose and O2Pulse may provide mechanistic insight into the cardiac reserve of the CA survivor following thoracic RT. METHODS/ STUDY POPULATION: Patients without a history of cardiovascular disease with a history of thoracic RT for CA treatment with significant incidental heart exposure ( $\geq 5$ Gray (Gy) to $\geq 10 \%$ of the heart volume) underwent treadmill CPET to determine cardiorespiratory fitness and cardiac magnetic resonance (CMR) imaging to quantify central hemodynamics and for myocardial tissue characterization. The mean cardiac radiation dose (MCRD) and \%volume of heart dose was determined from dose-volume histograms reflective of the dose contributions from all RT treatments for each patient. The oxygen pulse (milliliters $(\mathrm{mL})$ of $\mathrm{O} 2$ per heart beat) was determined by dividing the absolute VO2 by the HR (beats per minute, $\mathrm{bpm})$ at peak exercise and reported as \%-predicted values to account for age and gender differences. Data are reported as number (\%) or median (interquartile range). A stepwise multivariate linear regression model was created from significant univariate RT and CMR variables to determine independent predictors of \%O2Pulse. RESULTS/ ANTICIPATED RESULTS: Thirty patients (age $=63$ [57-67] years, 18 [60\%] female, 2.0 [0.1-28.7] years since completion of RT) underwent study procedures. The peak VO2 $=1376 \mathrm{~mL} \cdot \mathrm{min}-1(62 \%$ of predicted) and peak $\mathrm{HR}=150(122-164) \mathrm{bpm}$ resulted in a peak O2Pulse of $9.2 \mathrm{~mL} /$ beat $(82 \%$ of predicted). The $\mathrm{MCRD}=5.6$ [3.7-17.8] Gy was inversely associated with \%O2Pulse at univariate analysis $(\mathrm{R}=-0.514, \mathrm{p}<.01)$, but was not retained at multivariate analysis. The CMR-derived CO ([4.9 (4.09-5.90) Liters/minute], $\beta=+.374, \mathrm{p}<.01)$, CMR-extracellular volume ([ECV, 26.9 (24.8$29.2) \%], \beta=-.536, p<.01)$, and volume of the heart exposed to $\geq 30$ Gy $([2.5(0-15.0) \mathrm{Gy}],(\beta=-.345, \mathrm{p}=.01)$ were retained in the model $(\mathrm{R} 2=.709, \mathrm{~F}(3,19)=15.438, \mathrm{p}<.001)$ and were independent predictors of the \%O2Pulse. DISCUSSION/SIGNIFICANCE OF IMPACT: In patients with significant heart exposure following RT, $\% \mathrm{O} 2 \mathrm{Pulse}$ (a surrogate of stroke volume response to exercise) is inversely associated with cardiac radiation dose and is related to central hemodynamics (CO) and markers of diffuse fibrosis (ECV).
Scientometric Analysis of the Puerto Rico Clinical and Translational Research Consortium (PRCTRC) Research Publications, 2010-2017

Mariela Torres- Cintrón ${ }^{1}$, Carlamarie Noboa ${ }^{1}$, Mariela Lugo-Pico ${ }^{2}$, Lorena Gonzalez-Sepulveda², Naydi Perez, RCM-PRCTRC ${ }^{1}$, Zulmarie De Pedro- Serbia ${ }^{2}$ and Margarita Irizarry-Ramírez ${ }^{1}$

${ }^{1}$ University of Puerto Rico-Medical Sciences Campus and

${ }^{2}$ Puerto Rico Clinical and Translational Research Consortium

OBJECTIVES/SPECIFIC AIMS: The objective of this study was to evaluate the impact of the research and the scientific collaborations as shown in research publication supported by the PRCTRC. METHODS/STUDY POPULATION: Manuscripts published from 2010 to 2017 were retrieved from the Science Citation Index database. Our search criteria included manuscripts: 1) with the PRCTRC grant number and 2) with a PMCID number. Scientometric indicators including h-index (HI), average citation (AC), collaboration coefficient (CC), collaboration index (CI) and degree of collaboration (DC) were calculated using the Web of Science Platform and Stata software for statistical inquiry. Joinpoint was used to calculate the annual percent change (APC). RESULTS/ANTICIPATED RESULTS: A total of 316 publications were identified from 20102017 , with an average of 39.5 publications per year, and a total of 2,383 citing articles without self-citations. During this period a significant growth (APC $=\neg 15.3 \%, \mathrm{p}<0.05)$ of scientific production was observed. The overall $\mathrm{HI}$ was 28 , and the AC per item was 9.31. Regarding collaboration, the overall CC was 0.82 , the CI was 6.93, and the DC was 99.3. DISCUSSION/SIGNIFICANCE OF IMPACT: This study demonstrated that the PRCTRC scientific production increased statistically significantly. Application of the scientometric indicators allows the PRCTRC assess the research productivity and collaboration to design and implement interventions according to program experience and needs.

The CTSA Institutional website: A higher purpose - Researcher use of institutional Clinical and Translational Science Award (CTSA) website content to assess or promote NCATS CTSA Program Goals.

Barbara Ann Tafuto ${ }^{1}$

Rutgers University

OBJECTIVES/SPECIFIC AIMS: The objective of this research was to identify and evaluate published research articles that highlight the use of CTSA institutional websites as a research tool or data source for translational science research. METHODS/STUDY POPULATION: A multifaceted systematic search process was engaged for this literature review process using standard literature database searching, digital journal database searching, and pearl growing. All U.S. based studies and reports from 2006 through the present that addressed the application of websites of CTSA institutions for translational science purposes were included in this review. Identified articles were collected, organized, and analyzed using an excel spreadsheet. There were 2 different data collection and organization protocols, one for studies the other for reports. The first data collection protocol was for identified studies that used individual CTSA Institutional websites as a data source for a research topic. The organization processes for each relevant study article included a customized data extraction process that looked to identify 
a standard group of key elements from each study: (1) The study's NCATS CTSA Goal. (2) The type of data searched in the CTSA institutional website. (3) The number of CTSA institutional websites searched. (4) The number of sites that had the needed data. (6) The outcomes reported from the research. The second data collection protocol was for identified reports that referenced single CTSA Institutional websites as performing a specific translational informatics functionality either as a portal to Clinical and Translational Science Award tools and resources or as a direct information source. The organizational process for each relevant report article also included a customized data extraction process that looked to identify a standard group of key elements from each report: (1) NCATS / CTSA Goal (2) Tool or Functionality Promoted (3) Description (4) Website used as portal or direct tool. (5) Target Audience. RESULTS/ANTICIPATED RESULTS: The studies were summarized using the standard group of key elements identified for data extraction and summarized in a table. In 5 of the 6 studies, researchers relied on CTSA member individual website content to mine necessary data. One (1) of the studies employed a mixed methods approach to data acquisition and only relied on CTSA member individual website content for CTSA institutions that did not respond to a user survey. One (1) study used a survey to learn about CTSA website content rather than review the websites. In 5 of the 6 studies, researchers reviewed individual CTSA websites for the purposes of determining the number or percentages of CTSA institutions had specific data. One (1) study instead reviewed the individual websites to develop a broader picture of what the CTSA Consortium offered as a group. The percentage of CTSA websites that had the needed data of the researchers ranged from $32 \%$ to $100 \%$. The median and mean scores for CTSA websites having the needed data was $66 \%$ and $66.5 \%$ respectively. One study did not provide specific information for assessment. All 6 studies included research that fell within at least 2 categories of the 5 NCATS CTSA Goal topics. The category most investigated was translational research processes where 5 of the 6 studies investigated how CTSA websites looked to improve the quality and efficiency of translational research. Three (3) studies investigated how CTSA's cultivated and trained the clinical and translational science workforce. Two (2) studies investigated how CTSA's engaged patients and communities in the translational research process. Two (2) studies investigated how CTSA's promoted the integration of underserved populations. One (1) study investigated ways the CTSA's used their websites to advance the use of cutting-edge informatics. The outcomes reported included (1) the percentage CTSA individual websites that provided information regarding patient recruitment. (2) A list of generic services provided across the CTSA Individual website medium. (3) The number of CTSA individual website education and training programs. (4) The number and quality of informed consent forms presented online. (5) Investigational New Drug (IND) / Investigational Device Exemption (IDE) training methods for CTSA Investigators. (6) The percentage of KL2 Awards used by Child Health Investigators at CTSA Institutions. The reports $(\mathrm{rn}=9)$ were also summarized using the standard group of key elements identified for data extraction and summarized in a table. All six articles reported using their Institutional CTSA website as either a portal or a tool to promote clinical and translational science as outlined through NCATS goals. A CTSA website is used as a portal when it provides links to other sites, tools, or programs. A CTSA Website is used as a tool when it provides the functionality within its web design like providing an online application or database, or interactive training pages. In 8 of the 9 articles, authors reported on CTSA institutional website as either a translational informatics portal or providing informatics functionalities. Four (4) of the articles reported the use of their website for engagement, on either the collaborator or patient level, such as advocacy, education, or subject enrollment. Two (2) articles reported the use of their CTSA website for the cultivation and training of a clinical and translational science workforce. Four (4) articles reported on the use of their CTSA website for the purposes of increasing the quality and efficiency of translational research. None of the articles reported how their sites were used to promote the integration of underserved populations. All the reports identified a CTSA institutional website as a tool to leverage or disseminate CTSA capabilities and functionality. The access point and or warehousing of these capabilities was the CTSA institutional website. The target audience for these publications included researchers, clinical research administrators, IT programmers, community collaborators, and research subjects. The articles that reported on the use of CTSA institutional websites for clinical and translational functionality included topics such as: (1) the introduction of an informatics tool that searches clinical notes to identify clinical data for research. (2) the promotion of an online research subject advocacy program. (3) the introduction of an informatics tool portal that allows researchers flexible, efficient and effective means of collaboration and interaction with data. (4) the promotion of a team development project tool. (5) the introduction of a research participant registry and study promotion and education tool. (6) the promotion of an independent informatics tool registry that could connect to all CTSA websites. DISCUSSION/SIGNIFICANCE OF IMPACT: This research shows that CTSA institutional website functionality and content contributes to the CTSA body of research and the advancement NIH translational science goals.

\section{TL1 Team Approach to Using a Combination of Ganglioside 2 and 3 as an Immunoaffinity Target for Circulating Osteosarcoma Cell Detection}

Henrietta Fasanya ${ }^{1}$, Pablo Joaquin Dopico ${ }^{1}$, Zachary J. Yeager ${ }^{1}$, Hugh Fan ${ }^{1}$ and Dietmar W. Siemann ${ }^{1}$

${ }^{1}$ University Of Florida

OBJECTIVES/SPECIFIC AIMS: The objective of our collaboration is to develop a strong trans-disciplinary team consisting of microfluidics engineers, cancer biologists, and clinicians, to identify a universal marker to detect circulating osteosarcoma cells (COC) using microfluidic devices. Our goals are 3 fold: 1) Identify cell surface markers unique to osteosarcoma (OS) for COC isolation, 2) Develop a Geometrically Enhanced Mixing (GEM) device to isolate COCs, and 3) Evaluate the efficacy of GEM device to detect COCs in patients with OS. The long term goal of this collaboration is to utilize this cell detection approach to evaluate treatment efficacy and correlate the presence of circulating osteosarcoma cells with metastatic incidence. METHODS/STUDY POPULATION: In this phase of our study, we have identified an abundant and conserved cell surface marker across a panel of OS cell lines. Flow cytometry was used to evaluate the relative expression of Epithelial Cell Adhesion Molecule (EpCAM), and Ganglioside 2 or/and 3 (GD2/3) on a panel of OS cell lines. An antibody coated GEM microfluidic device is used to affirm the efficacy of GD2/3 to capture COCs. Further capture studies will be conducted using OS cell spiked blood samples. Analysis of variance (ANOVA) will be used to determine any significant difference in capture efficiency between EpCAM, GD2/3 cell surface markers. RESULTS/ANTICIPATED RESULTS: Our results demonstrate that EpCAM is not a suitable marker for COC 